\title{
SUPERCONDUCTING PROPERTIES AND PHASE SEPARATION EFFECTS IN SYSTEMS WITH LOCAL PAIRING
}

\author{
G. Pawlowski and S. Robaszkiewicz \\ Institute of Physics, A. Mickiewicz University \\ Umultowska 85, 61-614 Poznań
}

\begin{abstract}
We examine the phase separation effects in a class of models developed for description of superconductors with local electron pairing: (i) the extended attractive Hubbard model, (ii) the model of hard-core charged bosons on a lattice. We analyse the behaviour of various superfluid characteristics as well as the evolution of the phase diagrams with increasing external magnetic field.
\end{abstract}

PACS numbers: $74.20 .-\bar{z}, 05.30 . J p, 71.28 .+d$

We consider two effective models of superconductors with short coherence length [1-5]:

(i) the extended Hubbard model (EHM) with local attraction $(U<0)$, longer-range repulsion $\left(W_{i j}\right)$

$$
H_{\mathrm{I}}=\sum_{i j \sigma} t_{i j} c_{i \sigma}^{+} c_{j \sigma}+U \sum_{i} n_{i \uparrow} n_{i \downarrow}+\frac{1}{2} \sum_{i j \sigma \sigma^{\prime}} W_{i j} n_{i \sigma} n_{i \sigma^{\prime}}, \quad 0<n<2
$$

$t_{i j}$ is the transfer integral and $n=N_{\mathrm{e}} / N$ is the electron density per site;

(ii) the model of hard-core charged bosons on a lattice

$$
H_{\mathrm{II}}=\sum_{i j \sigma} J_{i j} b_{i}^{+} b_{j}+\sum_{i j} K_{i j} n_{i} n_{j}, \quad 0<\bar{n}<1,
$$

$b_{i j}^{(+)}$are the Pauli operators, $n_{i}=b_{i}^{+} b_{i}, J_{i j}$ is the transfer integral of electron pairs (hard core bosons with charge $2 e$ ) from site $i$ to $j, K_{i j}$ is the intersite density interaction between the pairs, $\bar{n} \equiv n / 2$ is the boson concentration per site.

Equation (2) is the minimum Hamiltonian for the local pair models of superconductivity. This model is appropriate for description of the large $|U| / t$ limit of the model (1) $[1,2]$ and in such case

$$
J_{i j}=2 t_{i j}^{2} /|U|, \quad K_{i j}=J_{i j}+2 W_{i j}, \quad \bar{n}=n / 2 .
$$

It also covers the case of the intersite pairing, the large bipolarons and magnetic bipolarons [1]. 
At half-filling both above models exhibit under definite conditions a SU(2) symmetry: the first one for $W_{i j}=0$, the second one for $K_{i j}-J_{i j}=0$ [3-5]. This SU(2) symmetry implies that the superconducting (SS) and charge ordered (CDW) ground states are degenerate and related by a rotation. Although departure from half-filling and (or) the inclusion of $W_{i j} \neq 0$ or $K_{i j}-J_{i j} \neq 0$ breaks this symmetry, its physical effects should still persist strongly and they are indicative of a new type of SU(2) superconductivity for these systems. The most important of these effects is a strong tendency of the considered systems toward phase separations. As we have already shown in our recent paper [5], the calculated ground state phase diagrams indicate possibility of existence of several different types of the phase separated (PS) states.

In this work we continue our studies [5] and report results concerning the behaviour of various superfluid characteristics as well as the evolution of the phase diagrams with increasing external magnetic field. In the analysis we used the broken symmetry HFA, for the model (1) [2b] and the MFA-RPA theory for the model (2) [2a]. The free energies of the homogeneous phases (SS, CDW-SS, CDW, NO) are determined in a standard way $[1,2,5]$, whereas the energy of the PS states is calculated from the expression

$$
E_{\mathrm{PS}}=m E\left(n_{+}\right)+(1-m) E\left(n_{-}\right), \quad m n_{+}+(1-m) n_{-}=n,
$$

where $E\left(n_{ \pm}\right)$is the value of the energy $E=\langle H\rangle / N$ at $n=n_{ \pm}$corresponding to the lowest energy homogeneous solution, $m$ is fraction of the system with a,charge density $n_{+},(1-m)$ is a fraction with a density $n_{-}\left(n_{+} \neq n_{-}\right)$, and $\partial E\left(n_{-}\right) / \partial n_{-}=$ $\left[E\left(n_{+}\right)-E\left(n_{-}\right)\right] /\left(n_{+}-n_{-}\right)$(Maxwell construction).

Depending on the particle concentration and interaction parameters the phase diagrams of the systems considered are found to consist of at least seven different states, i.e., normal (NO), singlet superconducting (SS), charge ordered (CDW), homogeneous CDW-SS (M), CDW-SS phase separated (PS1: domains of SS $\left(n_{-} \neq 1\right)$ and CDW $\left(n_{+}=1\right)$ ), CDW-NO phase separated (PS2: domains of NO $\left(n_{-} \neq 1\right)$ and CDW $\left.\left(n_{+}=1\right)\right)$, and the state of particle droplets (PS3: domains of NO $\left(n_{+}>1\right)$ and NO $\left.\left(n_{-}<1\right)\right)$.

For the model (1) with nearest neighbor ( $\mathrm{nn}$ ) interactions only one finds that the superconducting PS1 state is always more stable than the homogeneous M phase, except of $|U| / D \gg 1$, where they become energetically degenerated. The region of stability of PS1 state is reduced by both the next nn repulsion $\left(W_{2}>0\right)$ and the long-range Coulomb interactions $W_{\mathrm{LR}}$. The most favorite conditions for the appearance of the $\mathrm{M}$ phase are found in the strong coupling limit $(|U| / D \gg 1)$. In such case arbitrary weak $W_{2}$ (or $W_{\mathrm{LR}}$ ) stabilizes this phase with respect to the PS1, whereas in the opposite limit the $M$ phase can develop only the presence of substantial $W_{\mathrm{LR}}$ (Fig. 1) (calculations performed using a rectangular density of states for $\epsilon_{k}$ ). As we see, the inclusion of the PS states and longer-range Coulomb interactions into consideration substantially modifies the phase diagrams obtained assuming only homogeneous phases.

For the models considered the thermodynamic critical field at $T=0$ is calculated from the expression $H_{\mathrm{c}}{ }^{2} / 8 \pi=\left(E_{\mathrm{NS}}-E_{\mathrm{SS}}\right) / a^{3}$, where $E_{\mathrm{SS}}$ is the internal energy of the superconducting state which is stable for given values of the interaction parameters and concentration (i.e. SS, M or PS1) and $E_{\mathrm{NS}}$ corresponds to the 


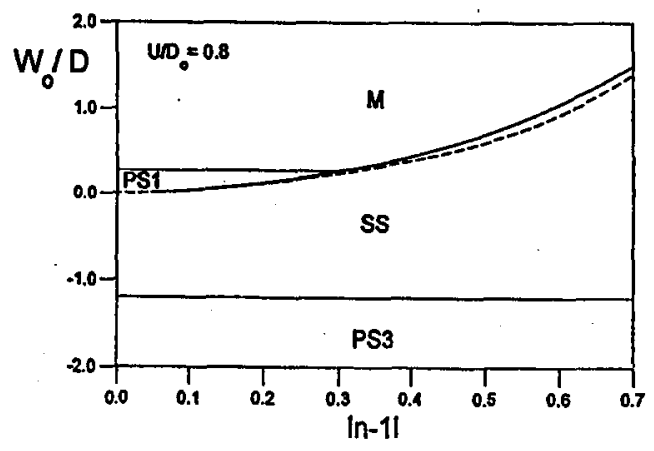

Fig. 1. Ground state phase diagram of the model (1) with $W_{i j}$ including the nn part $W_{1}\left(W_{0}=z_{1} W_{1}\right)$ and the long-range $W_{\mathrm{LR}}=\sum_{n>2} z_{n} W_{n}$, plotted as a function of $W_{0} / D$ and $|1-n|$ for $x=W_{\mathrm{LR}} / W_{0}=0.5$. Dashed line shows the boundary between the PS1 and SS for $x=0$. The M $\leftrightarrow$ SS transition is of 2nd order, whereas PS1 $\leftrightarrow$ M and PS $1 \leftrightarrow \mathrm{SS}$ are of 1st order. $|U| / D=0.8(D=z t, z-$ number of $\mathrm{nn})$.

lowest energy non-superconducting state for the same values of parameters (NO, CDW, PS2 or PS3), $a$ is the lattice constant.

The London penetration depth at $T=0$ in the local limit is given by

$$
\frac{1}{\lambda^{2}}=\frac{8 \pi e^{2}|t|}{h^{2} c^{2} a N} \sum_{k \sigma}\left\langle c_{k \sigma}^{+} c_{k \sigma}\right\rangle \cos k_{\alpha},
$$

and by

$$
\frac{1}{\lambda^{2}}=\frac{32 \pi e^{2}}{h^{2} c^{2} a N z} \sum_{k} J_{k}\left\langle b_{k}^{+} b_{k}\right\rangle,
$$

for the models (1) and (2), respectively $(\alpha=x, y, z)$. Using the values of $\lambda$ and $H_{\mathrm{c}}$ one is able to determine the Ginzburg-Landau length as $\xi_{\mathrm{GL}}=\Phi_{0} /\left(2 \pi \sqrt{2} \lambda H_{\mathrm{c}}\right)$ where $\Phi_{0}=h c / 2 e$, and obtain the estimations for $\kappa=\lambda / \xi_{\mathrm{GL}}$ and for the critical fields

$$
H_{\mathrm{c} 1} \approx \frac{\ln \kappa}{\kappa} H_{\mathrm{c}}, \quad H_{\mathrm{c} 2} \approx \frac{\Phi_{0}}{2 \pi \xi^{2}} .
$$

In Fig. 2 we present the plots of $H_{\mathrm{c}}, \lambda, \xi_{\mathrm{GL}}$ and the Ginzburg ratio $\kappa$ calculated for the attractive Hubbard model $\left(W_{i j}=0\right)$.

With increasing $|U|$ the $H_{c}^{2}$ increases exponentially for small values of $|U|$, then goes through a round maximum and it decreases as $t^{2} /|U|$ for large $|U|$. - The maximum is placed in the crossover regime (at $|U| / D \approx 1.4 \div 1.6$ ) and its position only slightly depends on the electron density. Notice that qualitatively the same $|U|$ dependence has been also predicted for $T_{\mathrm{c}}$ [1]. In Fig.2b we show the evolution of $\xi_{\mathrm{GL}}$ and $\kappa$ with $|U|$. With increasing $|U|$ the correlation length rapidly decreases at small $|U|\left(\xi_{\mathrm{GL}} \propto \exp (-|U| / D)\right.$, for $\left.|U| \ll D\right)$ and tends to a constant value, dependent on $n$, at large $|U|$. The increase in $\kappa$ with $|U|$ is exponential at small $|U|$, whereas in the opposite limit $\kappa$ becomes proportional to $\sqrt{|U| / t^{2}}$. A crossover between those two types of behavior takes place for intermediate values of $|U|(1<|U| / D<2)$. 

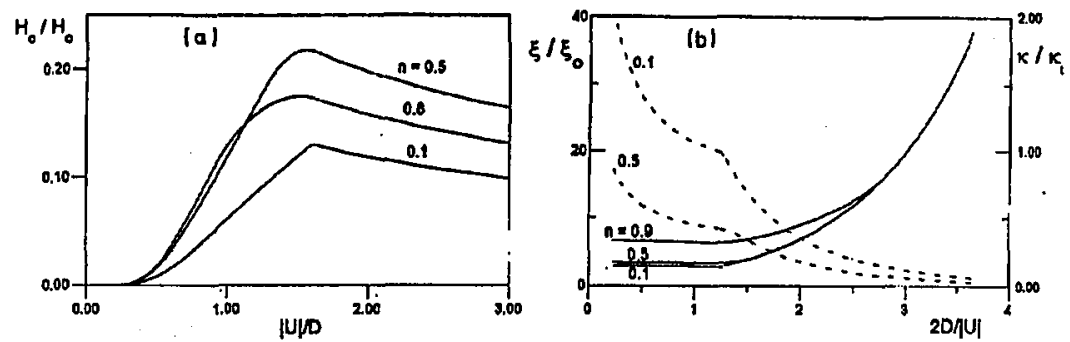

Fig. 2. Thermodynamical critical field $H_{\mathrm{c}}(T=0)(\mathrm{a})$ and the G-L coherence length $\xi$ (solid line) and the Ginzburg ratio $\kappa=\lambda / \xi$ (dashed line) (b) for the model (1), $W_{i j}=0$ as a function of $|U|$ for fixed $n, H_{0}=4.48 \times 10^{2}\left[z t[\mathrm{eV}] /\left(a_{\|}^{2} a_{\perp}\left[\AA^{3}\right]\right)\right]^{1 / 2}, \xi_{0}=a \sqrt{2 z}[\AA]$, $\kappa_{0}=73.3\left[a_{\perp}[\AA] /(t / 4[\mathrm{eV}])\right]^{1 / 2} / \xi_{0}$.
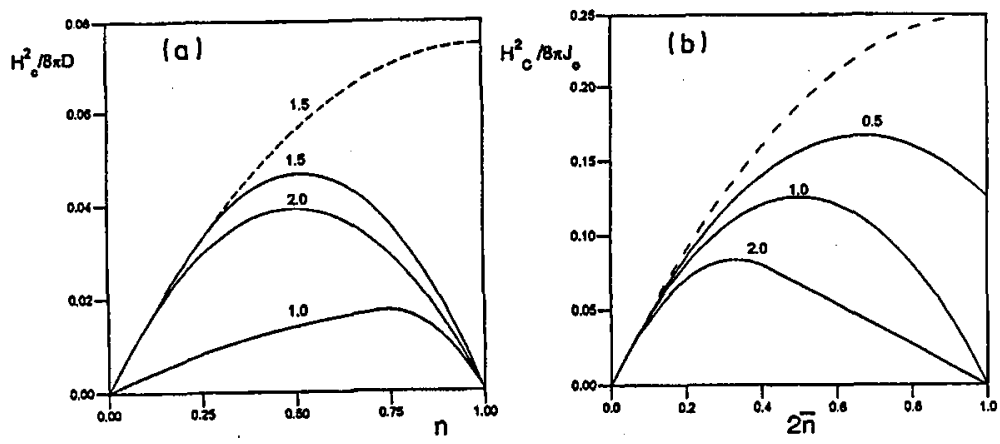

Fig. 3. Thermodynamical critical field $H_{c}^{2}$ as a function of $n:$ (a) $\operatorname{model}(1): U / D=1.0$, 1.5, $2.0\left(W_{i j}=0\right)$, (b) model (2): $K / J=0.5,1.0,2.0$.

As it follows from Fig. 3 in the high concentration limit (i.e. around half-fillings) the $H_{\mathrm{c}}$ of the systems considered can be much lower than that of a conventional, Abelian superconductor, which has only an U(1) symmetry. In the latter case there are no orderings competing with superconductivity and $H_{\mathrm{c}}{ }^{2} / 8 \pi=$ $\left(E_{\mathrm{NO}}-E_{\mathrm{SS}}\right) / a^{3}$. This quantity calculated for our models is shown in Fig. 3 by a dashed line.

By comparison of the Gibbs free energies $G(H)$ of all considered states we determined the evolution of the phase diagrams as a function of external magnetic field. The results indicate that with increasing magnetic field the systems can exhibit, depending on electron concentration and interaction parameters, either a single transition $\mathrm{SS} \rightarrow \mathrm{NO}, \mathrm{SS} \rightarrow \mathrm{PS} 2, \mathrm{PS} 1 \rightarrow \mathrm{PS} 2$, or a sequence of phase transitions, e.g. $\mathrm{M} \rightarrow \mathrm{PS} 1 \rightarrow \mathrm{PS} 2$ and $\mathrm{SS} \rightarrow \mathrm{PS} 1 \rightarrow \mathrm{PS} 2$. Examples of the obtained diagrams are shown in Fig. 4. Figure 4a is plotted for $|U| / D=1$ (intermediate coupling regime). In the large $|U|$ limit the corresponding diagrams for the model (1) with $W_{i j}=0$ take the form analogous to that found for the model (2) at $K / J=1$ (Fig. $4 \mathrm{~b}$ ). 

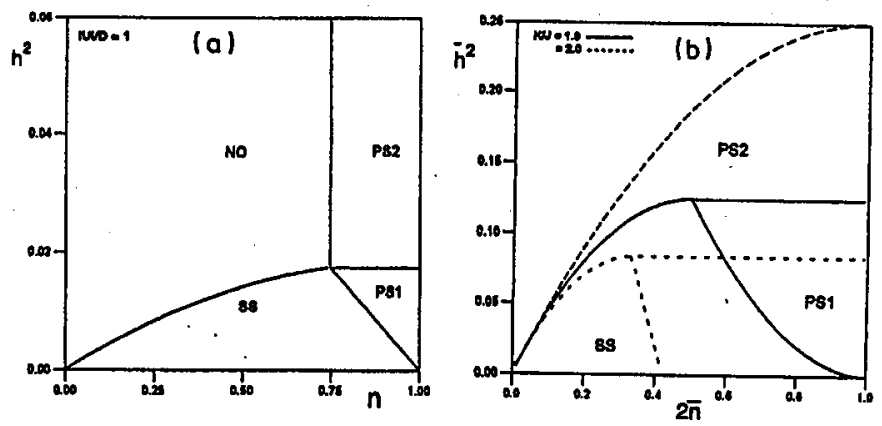

Fig. 4. Ground state phase diagrams in the presence of magnetic field (a) for model (1): $|U| / D=1, W=0$, (b) for model (2): $K / J=1.0$ (solid) and $=2.0$ (dashed). $h^{2}=$ $H^{2} /(8 \pi D), \bar{h}^{2}=H^{2} /(8 \pi J)$.

\section{Acknowledgments}

We would like to thank R. Micnas and T. Kostyrko for discussions. This work was supported by the Committee for Scientific Research, projects 2PO3B104 1-1 and 2PO3B0570.9.

\section{References}

[1] R. Micnas, J. Ranninger, S. Robaszkiewicz, Rev. Mod. Phys. 62, 113 (1990).

[2] S. Robaszkiewicz, R. Micnas, K.A. Chao, (a) Phys. Rev. B 23, 1441 (1981); (b) 24, 4018 (1981); 26, 3915 (1982).

[3] S. Zhang, Phys. Rev. Lett. 65, 120 (1990).

[4] S. Soni, J.S. Thakur, Phys. Rev. B 48, 12917 (1993).

[5] S. Robaszkiewicz, G. Pawłowski, Mol. Phys. Rep. 12, 205 (1995); idem, to be published. 